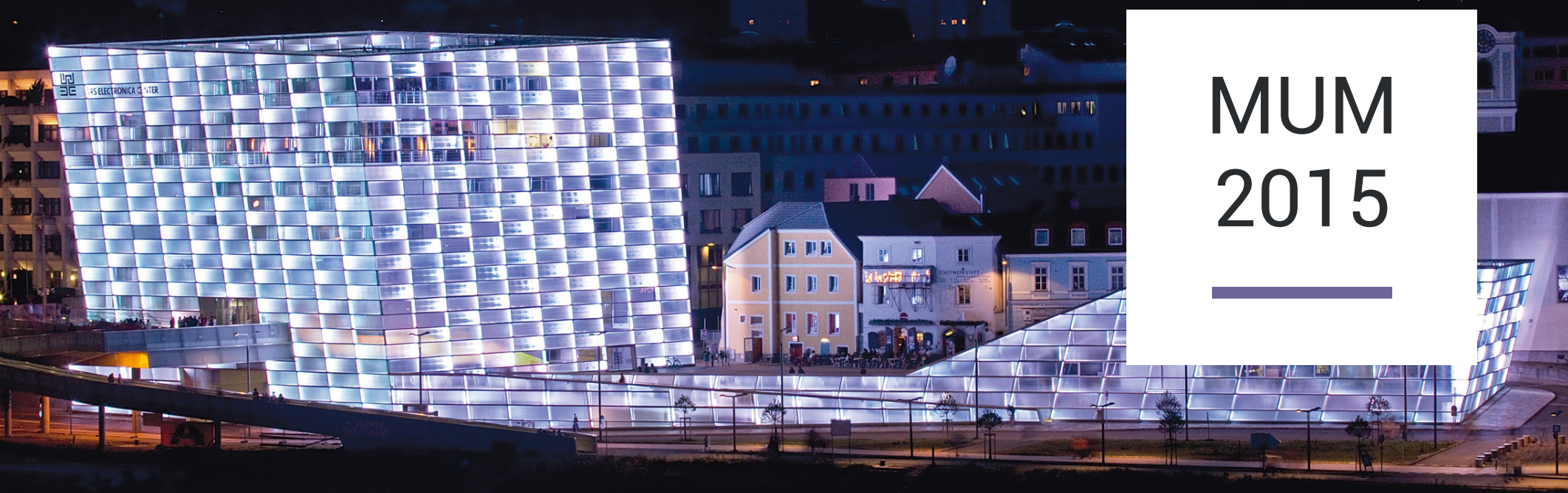

14th International Conference on

\title{
MOBILE AND UBIQUITOUS MULTIMEDIA
}

\section{Proceedings}

Nov 30 - Dec 2, 2015 | Linz, Austria

\section{Editors}

Clemens Holzmann

René Mayrhofer

Jonna Häkkilä

Enrico Rukzio

Michael Roland

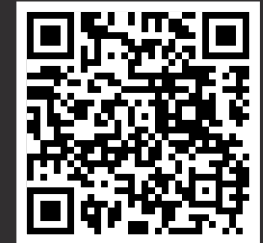

www.mum-conf.org/2015
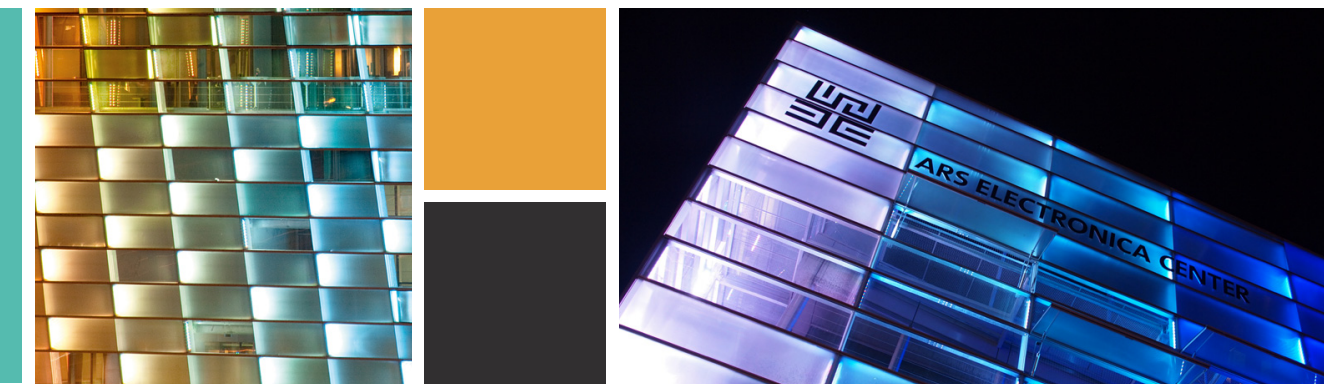
Clemens Holzmann, René Mayrhofer, Jonna Häkkilä, Enrico Rukzio, Michael Roland (eds.)

\section{MUM 2015}

\section{Proceedings of the \\ 14th International Conference on Mobile and Ubiquitous Multimedia}

November 30 - December 2, 2015

Linz, Austria
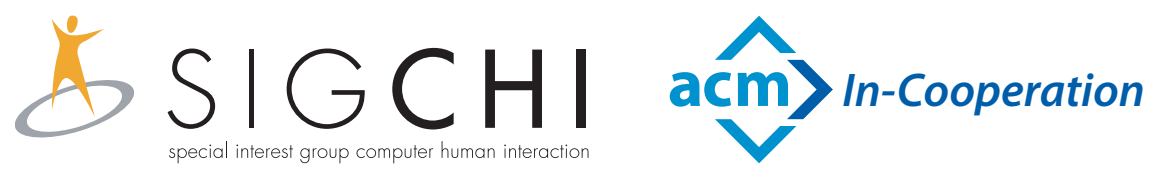


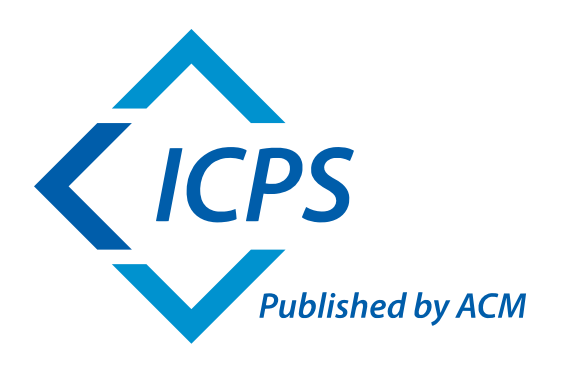

\section{The Association for Computing Machinery 2 Penn Plaza, Suite 701 New York New York 10121-0701}

\section{ACM COPYRIGHT NOTICE}

\section{Copyright (c) 2015 by the Association for Computing Machinery, Inc.}

Permission to make digital or hard copies of part or all of this work for personal or classroom use is granted without fee provided that copies are not made or distributed for profit or commercial advantage and that copies bear this notice and the full citation on the first page. Copyrights for components of this work owned by others than ACM must be honored. Abstracting with credit is permitted. To copy otherwise, to republish, to post on servers, or to redistribute to lists, requires prior specific permission and/or a fee. Request permissions from Publications Dept., ACM, Inc., fax +1-212-869-0481, or permissions@acm.org.

For other copying of articles that carry a code at the bottom of the first or last page, copying is permitted provided that the per-copy fee indicated in the code is paid through the Copyright Clearance Center, 222 Rosewood Drive, Danvers, MA 01923, tel +1-978-750-8400, fax +1-978-750-4470.

ACM ISBN: 978-1-4503-3605-5 


\section{Preface}

On behalf of the organizing committee for MUM 2015, we would like to welcome you to this 14th edition of the International Conference on Mobile and Ubiquitous Multimedia, held at the Ars Electronica Center in Linz, Austria from November 30 to December 2, 2015. The MUM conference series is a distinguished forum for advances in research and technologies that drives innovation in mobile and multimedia systems, applications, and services. At MUM academics and practitioners gather to discuss challenges and achievements from diverse perspectives, in a comfortable and effective single track conference format.

This year we received 89 full and short research paper submissions surrounding these topics. A rigorous review process followed where each paper received at least three reviews from international experts. After this review process, 25 full papers and 8 short papers were selected to appear in these proceedings and to be presented at the conference. This results in an acceptance rate of 37\% for 2015. The topics in the paper program are organized in the following sessions: media and applications; studies and findings; detecting, classifying, and recognizing; specific application areas; specific user groups; wearable and mobile interaction; augmented reality; and designing interactions. We hope that this selection of papers will form the basis of lively discussions at the conference as well as new cooperations leading to future research.

The program committee awards best paper awards to the authors of two papers, "Wearable Technology as a Solution for Workplace Safety" and "Effects of Camera Position and Media Type on Lifelogging Images", along with three further honorable mentions. These awards and honorable mentions were selected due to their very positive reviews and represent a special recognition of excellence.

In addition to the regular paper submissions, MUM 2015 is happy to feature a poster and a demo track again, with 15 accepted posters and 5 accepted demos. This year we introduce a video track with 3 accepted videos as well as a doctoral school with 3 accepted submissions. Altogether, 26 submissions to adjunct categories were accepted for publication in addition to the 33 full and short papers. We encouraged authors of adjunct submissions to submit to multiple categories (i.e. poster, demo, video, and doctoral school). Moreover, we encouraged authors of accepted full and short papers to also submit a demo or video for their papers. Therefore, one paper from the conference proceedings may be represented in multiple presentation categories.

In addition, we are pleased to have two keynotes this year. The opening keynote, "Eye Movement for Multimodal and Ubiquitous Interaction", is held by Hans Gellersen, Professor at Lancaster University, United Kingdom, and the closing keynote, "How ubiquitous and pervasive do computer systems have to become before we really start to care about the users?", is held by Gerfried Stocker, Artistic Director at Ars Electronica, Austria. 
There are a number of people we want to acknowledge, whose help greatly simplified the organization of this conference. Firstly, we acknowledge the support of the University of Applied Sciences Upper Austria, the Johannes Kepler University Linz, and the Ars Electronica Center. Secondly, we warmly thank all the members of the program committee and the additional reviewers for their helpful feedback and constructive criticisms. We would also like to thank all the student volunteers and other persons that contributed to the organization of the conference. Finally, we thank all the authors and participants of this event for sharing new insights, concepts and results on mobile and ubiquitous multimedia research.

We welcome you to enjoy MUM 2015 in Linz!

Clemens Holzmann
MUM 2015 General Chair
University of Applied Sciences Upper Austria
Hagenberg, Austria
Jonna Häkkilä
MUM 2015 Program Chair
University of Lapland
Rovaniemi, Finland

René Mayrhofer MUM 2015 General Chair Johannes Kepler University Linz Linz, Austria

Enrico Rukzio
MUM 2015 Program Chair
University of Ulm
Ulm, Germany




\section{Committees and Reviewers}

\section{Organizing Committee}

\section{General Chairs}

Clemens Holzmann

University of Applied Sciences Upper Austria, Hagenberg, Austria

René Mayrhofer

Johannes Kepler University Linz, Linz, Austria

\section{Program Chairs}

\section{Jonna Häkkilä}

University of Lapland, Rovaniemi, Finland

Enrico Rukzio

University of Ulm, Ulm, Germany

\section{Demonstration Chairs}

Vassilis Kostakos

University of Oulu, Oulu, Finland

Matthias Kranz

University of Passau, Passau, Germany

\section{Poster Chairs}

Bernd Ludwig

University of Regensburg, Regensburg, Germany

Kristof Van Laerhoven

University of Freiburg, Freiburg, Germany

\section{Video Chairs}

Christopher Lindinger

Ars Electronica Futurelab, Linz, Austria 


\section{Dominik Schmidt}

Cliqz GmbH, Munich, Germany

\section{Doctoral School Chairs}

\section{Hans Gellersen}

Lancaster University, Lancaster, UK

Michael Haller

University of Applied Sciences Upper Austria, Hagenberg, Austria

\section{Publicity Chairs}

Andreas Riener

Ingolstadt University of Applied Sciences, Ingolstadt, Germany

Florian Schaub

Carnegie Mellon University, Pittsburgh, PA, USA

\section{Publications Chair}

\section{Michael Roland}

University of Applied Sciences Upper Austria, Hagenberg, Austria

\section{Local Arrangement Chairs}

\section{Severina Lachmair-Kehayova}

Johannes Kepler University Linz, Linz, Austria

Nicola Spitzer

University of Applied Sciences Upper Austria, Linz, Austria

\section{Web Chair}

\section{Elisabeth Hackner}

University of Applied Sciences Upper Austria, Hagenberg, Austria 


\section{Program Committee}

Florian Alt, Ludwig-Maximilians-Universität München, Munich, Germany

Sebastian Boring, University of Copenhagen, Copenhagen, Denmark

Ashley Colley, University of Oulu, Oulu, Finland

Christos Efstratiou, University of Kent, Kent, UK

Parisa Eslambolchilar, Swansea University, Swansea, UK

Jens Grubert, Graz University of Technology, Graz, Austria

Jonathan Hook, University of York, York, UK

Wolfgang Hürst, Utrecht University, Utrecht, The Netherlands

Marko Jurmu, University of Oulu, Oulu, Finland

Johan Kildal, Nokia Technologies, Espoo, Finland

Timo Koskela, University of Oulu, Oulu, Finland

Hannu Kukka, University of Oulu, Oulu, Finland

Markus Löchtefeld, DFKI, Saarbrücken, Germany

Florian Michahelles, Siemens, Berkeley, CA, USA

Cosmin Munteanu, University of Toronto Mississauga, Mississauga, Ontario, Canada

Timo Ojala, University of Oulu, Oulu, Finland

Thomas Olsson, Tampere University of Technology, Tampere, Finland

Fabio Paternò, CNR-ISTI, HIIS Laboratory, Pisa, Italy

Martin Pielot, Telefonica Research, Barcelona, Spain

Markus Rittenbruch, Queensland University of Technology, Brisbane, Australia

Simon Robinson, Swansea University, Swansea, UK

Michael Rohs, University of Hannover, Hannover, Germany

George Roussos, University of London, London, UK

Alireza Sahami, Yahoo! Labs, Sunnyvale, CA, USA

Florian Schaub, Carnegie Mellon University, Pittsburgh, PA, USA

Johannes Schöning, Hasselt University, Hasselt, Belgium

Tsutomu Terada, Kobe University, Kobe, Japan

Kristof van Laerhoven, University of Freiburg, Freiburg, Germany

Katrin Wolf, University of Stuttgart, Stuttgart, Germany 


\section{Contributing Reviewers}

\author{
Ismo Alakärppä \\ Hazim Almuhimedi \\ Irina Anastasiu \\ Sergey Andreev \\ Kamyar Ardekani \\ Daniel Ashbrook \\ Rebecca Balebako \\ Frank Bentley \\ Jelmer Borst \\ Keith Cheverst \\ Rob Comber \\ Paulo de Barros \\ Imar de Vries \\ Jingjing Deng \\ Tilman Dingler \\ Paul Dunphy \\ Golnoosh Farnadi \\ Daniel Filonik \\ Rachel Franz \\ Daniel Gallardo \\ Giuseppe Ghiani \\ Joseph Grafsgaard \\ Erkki Harjula \\ Arto Heikkinen \\ Wilko Heuten \\ Frank Honold \\ Steven Houben \\ Pertti Huuskonen \\ Denis Kalkofen \\ Mohamed Khamis \\ Tomi Knuutila \\ Markus Krause \\ Stefan Kueppers \\ Christian Lander \\ Manfred Lau \\ Stephen Lindsay \\ Marco Manca \\ Angelos Marnerides \\ Abhinav Mehrotra \\ Nemanja Memarovic \\ Aske Mottelson \\ Niko Mäkitalo \\ Timothy Neate
}

Paula Alavesa

Ofra Amir

Mads Schaarup Andersen

Jim Ang

Bon Adriel Aseniero

Matthias Baldauf

Alessandro Andrea Bendinelli

Niels Van Berkel

Richard Byrne

Sarah Clinch

Ioannis Daskalopoulos

Michiel de Lange

Carrie Demmans Epp

Manuel Dietrich

Jared Donovan

Ayman El Hajjar

Denzil Ferreira

Zachary Flamig

Markus Funk

Sven Gehring

Jorge Goncalves

Cathal Gurrin

Andreas Hartl

Niels Henze

Gerold Hölzl

Simo Hosio

Jukka Huhtamäki

Naoya Isoyama

Eiman Kanjo

Pascal Knierim

Robert Kovacs

Mareike Kritzler

Tanesh Kumar

Tobias Langlotz

Pascal Lessel

Andrés Lucero

Anders Markussen

Paul Matthews

Tenager Mekonnen

Giulio Mori

Kazuya Murao

Takashi Nagamatsu

James Nicholson 
Sofia Nikitaki

Tarik Ozkul

Minna Pakanen

Joy Piontak

Matti Pouke

Banda Ramadan

Thomas Reitmaier

Yvonne Rogers

Carmen Santoro

Stefan Schneegass

Ronald Schröter

Hartmut Seichter

Adalberto Simeone

Madeline Smith

Pieter Jan Stappers

Paul Strohmeier

Chiew Seng Sean Tan

Robyn Taylor

Tuomas Vaittinen

Nervo Xavier Verdezoto

Vasilis Vlachokyriakos

Robert Walter

Benjamin Weiss

Frederik Wiehr

Matthias Wille

Stephan Winter

Xianghua Xie

Johanna Ylipulli

Guoying Zhao
Jarno Ojala

Susanna Paasovaara

Bastian Pfleging

Gerald Pirkl

Kiran Rachuri

Raf Ramakers

Katja Rogers

Nina Rosa

Antonio Giovanni Schiavone

Philipp M. Scholl

Valentin Schwind

Sajad Shirali-Shahreza

Konstantinos Sirlantzis

Alessandro Soro

Tobias Stockinger

Iván Sánchez

Markus Tatzgern

Daniel Ullrich

Elba Del Carmen Valderrama Bahamondez

Jo Vermeulen

Dhaval Vyas

Dominik Weber

Dirk Wenig

Jason Wiese

Julie Williamson

Robert Xiao

Keiko Yamamoto

Yanxia Zhang 


\section{Table of Contents}

Preface

Committees and Reviewers

Organizing Committee . . . . . . . . . . . . . . . . . . . . . v v

Program Committee . . . . . . . . . . . . . . . . . . . . . vii

Contributing Reviewers . . . . . . . . . . . . . . . . . . . viii

\section{Proceedings of MUM 2015}

\section{Keynote TALKS}

Eye Movement for Multimodal and Ubiquitous Interaction . . . . . . . . . . . . . xxi Hans Gellersen

How ubiquitous and pervasive do computer systems have to become before we really start to care about the users? . . . . . . . . . . . . . . . . . . . . xxii Gerfried Stocker

\section{Media AND Applications}

Pick Up and Play: Understanding Tangibility for Cloud Media

Donald Mcmillan, Barry Brown, Abigail Sellen, Siân Lindley, and Roy Martens

On-the-fly Entity Resolution from Distributed Social Media Sources for Mobile Search and

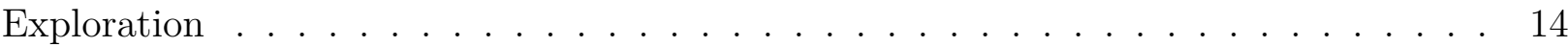

Bernd Opitz, Timo Sztyler, Michael Jess, Florian Knip, Christian Bikar, Bernd Pfister, and Ansgar Scherp

What's the deal with privacy apps? A comprehensive exploration of user perception and usability

Hala Assal, Stephanie Hurtado, Ahsan Imran, and Sonia Chiasson

Redefining the Fundamentals of Photography with Cooperative Photography . . . . . . . 37 James Wen and Ayça Ünlüer

\section{STUDIES AND FINDINGS}

User Experience of Digital News: Two Semi-long Term Field Studies 
Street Art Gangs: Location Based Hybrid Reality Game . . . . . . . . . . . . . . . . . . . 64 Paula Alavesa and Timo Ojala

Show Me Your Phone, I Will Tell You Who Your Friends Are: Analyzing Smartphone Data To Identify Social Relationships . . . . . . . . . . . . . . . . . . . . . . . . . . 75 Delphine Reinhardt, Franziska Engelmann, Andrey Moerov, and Matthias Hollick

Exploring Touch Interaction Methods for Image Segmentation on Mobile Devices .. . . 84 Christoph Korinke, Nils Steffen Worzyk, and Susanne Boll

\section{Detecting, Classifying, and Recognizing}

Detecting Human Encounters from WiFi Radio Signals . . . . . . . . . . . . . . . . . . 97 Geert Vanderhulst, Afra Mashhadi, Marzieh Dashti, and Fahim Kawsar

Happy and Agreeable? Multi-Label Classification of Impressions in Social Video . . . . . . 109 Gilberto Chávez-Martínez, Salvador Ruiz-Correa, and Daniel Gatica-Perez

Assessing Activity Recognition Feedback in Long-term Psychology Trials . . . . . . . . . . 121 Manuel Dietrich, Eugen Berlin, and Kristof van Laerhoven

Towards Device-to-User Authentication: Protecting Against Phishing Hardware by Ensuring Mobile Device Authenticity using Vibration Patterns (Short Paper) . . . . . . . . . 131 Rainhard Dieter Findling and René Mayrhofer

\section{Specific Application Areas}

Triage Training System: Adjusting the Difficulty Level According to User Proficiency . . . 139 Yuki Tayama, Ryuga Kato, and Ken-ichi Okada

TwitSoccer - Knowledge-based Crowd-sourcing of live Soccer Events (Short Paper) . . . . . 148 Markus Löchtefeld, Christian Jäckel, and Antonio Krüger

'Weather With You': Evaluating Report Reliability in Weather Crowdsourcing .... . 152 Evangelos Niforatos, Athanasios Vourvopoulos, and Marc Langheinrich

Comic-Crowd: Interactive Comic Creation that Supports Multiple Storylines, Visualizations, and Platforms . . . . . . . . . . . . . . . . . 163 Hiroaki Tobita

\section{SPECIFIC USER GROUPS}

A Wearable and Mobile Intervention Delivery System for Individuals with Panic Disorder 175 Luis Cruz, Jonathan Rubin, Rui Abreu, Shane Ahern, Hoda Eldardiry, and Daniel G. Bobrow

Help Radar: Ubiquitous Assistance for Newly Arrived Immigrants . . . . . . . . . . . . . 183 Stephanie Schwarz, Estefania Palacio Salazar, Jan Bobeth, Nicoletta Bersia, and Manfred Tscheligi

Enabling Accessibility through Multimodality? Interaction Modality Choices of Older Adults (Short Paper) . . . . . . . . . . . . . . . . . . . . 195 Julia Himmelsbach, Markus Garschall, Sebastian Egger, Susanne Steffek, and Manfred Tscheligi

Localization of Storyboards for Cross-Cultural User Studies . . . . . . . . . . . . . . . . . 200 Tanja Walsh, Helen Petrie, and Anqi Zhang 


\section{Wearable AND Mobile INTERACTION}

Wearable Technology as a Solution for Workplace Safety (Short Paper) . . . . . . . . . . . . 213 Mareike Kritzler, Martin Bäckman, Anders Tenfält, and Florian Michahelles

WristRotate - A Personalized Motion Gesture Delimiter for Wrist-Worn Devices (Short Paper) . . . . . . . . . . . . . . . . . . . . 218

Frederic Kerber, Philipp Schardt, and Markus Löchtefeld

Design Probes Study on User Perceptions of a Smart Glasses Concept . . . . . . . . . . 223 Jonna Häkkilä, Farnaz Vahabpour, Ashley Colley, Jani Väyrynen, and Timo Koskela

Effects of Camera Position and Media Type on Lifelogging Images

Katrin Wolf, Yomna Abdelrahman, David Schmid, Tilman Dingler, and Albrecht Schmidt

Detecting Users Handedness for Ergonomic Adaptation of Mobile User Interfaces (Short Paper) . . . . . . . . . . . . . . . . . . . . . . 245 Markus Löchtefeld, Philipp Schardt, Antonio Krüger, and Sebastian Boring

\section{Augmented Reality}

A Benchmark for Interactive Augmented Reality Instructions for Assembly Tasks (Short Paper)

Markus Funk, Thomas Kosch, Scott W. Greenwald, and Albrecht Schmidt

Augmenting Food with Information _ . . . . . . . . . . . . . . . . . 258

Niels Henze, Thomas Olsson, Stefan Schneegass, Alireza Sahami Shirazi, and Kaisa Väänänen-Vainio-Mattila

Deriving Design Guidelines for Ambient Light Systems ～. . . . . . . . . . . . . . . . . . 267

Andrii Matviienko, Vanessa Cobus, Heiko Müller, Jutta Fortmann, Andreas Löcken, Susanne Boll, Maria Rauschen-

berger, Janko Timmermann, Christoph Trappe, and Wilko Heuten

Influence of Temporal Delay and Display Update Rate in an Augmented Reality Application Scenario . . . . . . . . . . . . . . . . . . . . . 278 Ming Li, Katrin Arning, Luisa Vervier, Martina Ziefle, and Leif Kobbelt

\section{DESIGNING INTERACTIONS}

Building Interactive Experiences Block by Block . . . . . . . . . . . . . . . . . . . . . . 289 Rossana Santos and Nuno Correia

De+re: A Design Concept For Provoking Meaningful Interactive Experiences . . . . . . . 302 Florian Güldenpfennig and Geraldine Fitzpatrick

Authoring Context-dependent Cross-device User Interfaces based on Trigger/Action Rules 313 Giuseppe Ghiani, Marco Manca, and Fabio Paternò

Opportunistic At-Glance Information Acquisition on Interactive Public Displays (Short Paper)

Hannu Kukka, Jorge Goncalves, Alexander Samodelkin, and Timo Ojala

\section{POSTER TRACK}

A Smartphone Application for Voice Browsing RFID Smart Shelves . . . . . . . . . . . . . 331 Kamruddin Nur, Zulqarnain Rashid, and Rafael Pous 
Destmaster - Improved Destination Input System For The UR-Walking Application . . . . 337 Stefan Covaci, Maximilian Frick, Florian Krämer, and Julian Pörsch

Emotion-based Music Recommendation using Supervised Learning . . . . . . . . . . . . . 341 Karl-Arnold Bodarwé, Jenny Noack, and Philipp Jean-Jacques

FROY: Exploring Sentiment-Based Movie Recommendations ～. . . . . . . . . . . . . . . 345 Philip Gaag, Daniel Granvogl, Robert Jackermeier, Florian Ludwig, Johannes Rosenlöhner, and Alexander Uitz

GRASPhere: A Prototype to Augment Indirect Touch with Grasping Gestures . . . . . . 350 Dorin-Mircea Popovici, Radu-Daniel Vatavu, and Mihai Polceanu

InfoFinder: Just-in-Time Information Interface from the Combination of an HWD with a Smartwatch . . . . . . . . . . . . . . . . . . 355 Jianming Wu and Tomonori Yazaki

Nombot - Simplify Food Tracking . . . . . . . . . . . . . . . . . . . . . 360 Bettina Graf, Maike Krüger, Felix Müller, Alexander Ruhland, and Andrea Zech

Collaborative Landmark Mining With A Gamification Approach . . . . . . . . . . . . . . 364 Florian Bockes, Laura Edel, Matthias Ferstl, and Andreas Schmid

Woody: A Location-based Smartphone Game to Increase Children's Outdoor Activities in Urban Environments . . . . . . . . . . . . . . . . . . 368 Paul Spiesberger, Florian Jungwirth, Christoph Wöss, Stefan Bachl, Johannes Harms, and Thomas Grechenig

Mobile for Older Adults: Towards Designing Multimodal Interaction . . . . . . . . . . . 373 Michela Ferron, Nadia Mana, and Ornella Mich

Landmark Mining on a Smartwatch Using Speech Recognition . . . . . . . . . . . . . . . 379 Jana Helgath, Simon Provinsky, and Timo Schaschek

Usability Requirements for Mobile Accessibility: A Study on the Vision Impairment . . . . 384 Clauirton Siebra, Tatiana Gouveia, Jefte Macedo, Walter Correia, Marcelo Penha, Fabio Silva, Andre Santos, Marcelo Anjos, and Fabiana Florentin

UI-CAT: Calculating User Interface Complexity Metrics for Mobile Applications . . . . . . 390 Andreas Riegler and Clemens Holzmann

Augmented Reality-Based Training of the PCB Assembly Process . . . . . . . . . . . . . . 395 Jürgen Hahn, Bernd Ludwig, and Christian Wolff

Understanding Usage Control Requirements in Pervasive Memory Augmentation Systems 400 Agon Bexheti and Marc Langheinrich

\section{Demo AND Video Tracks}

Interaction Visualization and Analysis in Automation Industry . . . . . . . . . . . . . . 407 Christian Grossauer, Clemens Holzmann, Dustin Steiner, and Andreas Guetz

MoCo Motion: Integrating Mobile Devices into a Multiplayer Floor-based Gaming Environment . . . . . . . . . . . . . . . . . . . . . 412 Andreas Friedl, Jeremiah Diephuis, Georgi Kostov, and Otto Naderer

Gary: Combining Speech Synthesis and Eye Tracking to Support Struggling Readers . . 417 Gianluca Schiavo, Simonetta Osler, Nadia Mana, and Ornella Mich 
Exploring AR Poster as an Interface to Personal Health Data . . . . . . . . . . . . . . . . 422 Juho Rantakari, Ashley Colley, and Jonna Häkkilä

Sauberes Wiesbaden App: Introducing Mobile E-Participation for a Clean City Project in

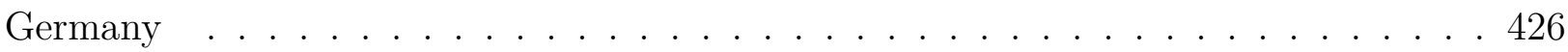
Stephan Böhm, Bodo Igler, Roberto Morales, Frank Sand, and Attila Ertan

\section{DOCTORAL SCHOOL}

Active Office: Designing for Physical Activity in Digital Workplaces . . . . . . . . . . . 433 Kathrin Probst

Collaborative Security Code Review . . . . . . . . . . . . . . . . . . . . . . . . 439 Hala Assal

The Mind Behind the Glass: Human Factors in the Design of Collaborative AR Environments . . . . . . . . . . . . . . . . . . . . . 445 Marion Koelle and Matthias Kranz

\section{Author Index}




\section{Proceedings of the}

\section{4th International Conference on Mobile and Ubiquitous Multimedia}




\section{MUM 2015 \\ Keynote Talks}




\title{
Eye Movement for Multimodal and Ubiquitous Interaction \\ (Opening Keynote)
}

\author{
Hans Gellersen \\ Professor of Interactive Systems \\ Lancaster University \\ School of Computing and Communications \\ Lancaster, UK
}

\begin{abstract}
Ubiquitous computing aspires to support human activity seamlessly with interactive technologies. This involves the proliferation of digital devices of different form and function in our environments, and the appropriation of diverse sensing modalities to observe activity, capture context, and provide natural interfaces to users. Among the many ways in which human activity can be observed, eye tracking has received only limited attention in ubiquitous computing. However, eye movement is a compelling source of information, as the eyes are where the action is; I will thus argue that eye movement can be leveraged in novel and advanced ways for multimodal and ubiquitous interaction.
\end{abstract}

Eye tracking has a long history and its use for human-computer interaction predates the ubiquitous computing era. While the technology has been maturing and become affordable for widespread use, there has not been much innovation in the use of eye gaze for interaction. For over 25 years, gaze pointing has remained the prevailing usage paradigm, although it overloads the sensory role of the eyes with a control function. In this talk I will present work that explores new ways of using eye movement for interaction. I will discuss gaze and touch: how hands and eyes can naturally work together; gaze and motion: how the natural gaze-following of moving stimuli enables new types of interface; and gaze and games: how gaze can be social and fun.

\section{ABOUT THE KEYNOTE SPEAKER}

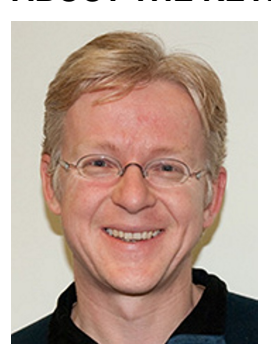

Hans Gellersen is Professor of Interactive Systems at Lancaster University. Hans' research interest is in sensors and devices for ubiquitous computing and human-computer interaction. He has worked on systems that blend physical and digital interaction, methods that infer context and human activity, and techniques for spontaneous interaction across devices. In recent work he is focusing on eye movement as a source of context information and modality for interaction. Hans was involved in the foundation of the UbiComp conference series, and serves as Associate Editor of ACM TOCHI and the Journal on Personal and Ubiquitous Computing (PUC). Hans has held his chair position at Lancaster since 2001. Previously he was affiliated with the University of Karlsruhe in Germany, where he received his $\mathrm{PhD}$ in Computer Science in 1996. 


\title{
How ubiquitous and pervasive do computer systems have to become before we really start to care about the users?
}

\author{
(Closing Keynote)
}

\author{
Gerfried Stocker \\ Artistic Director \\ Ars Electronica Linz GmbH \\ Linz, Austria
}

\begin{abstract}
In 1994 the Ars Electronica Festival was dedicated to "Intelligent Ambiences" and one of the pioneers of what we call nowadays ubiquitous and mobile computing, Rich Gold from the famous Xerox Parc Laboratories titled his lecture for the festivals symposium: "How smart does our bed have to be before we are afraid to sleep in it?". A wonderful and forward thinking title, considering that this was more than 20 years ago. A time where most of the concepts for such a future of computing were still speculative and many of them sounded as funny as this title. But now it's here or at least almost here. We are doing great in the technological developments towards it but when it comes to the social and cultural challenges, it seems we didn't learn much in the last two decades. So how ubiquitous and mobile can our digital world become at all, if we always just look at the technology behind it? What, if people really get afraid of these systems which we are preparing and implementing right now. And what can we do to get it right?
\end{abstract}

\section{ABOUT THE KEYNOTE SPEAKER}

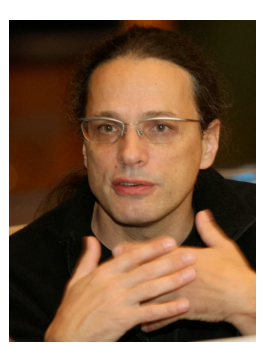

Gerfried Stocker is a media artist and telecommunications engineer. In 1991, he founded $\mathrm{x}$-space, a team formed to carry out interdisciplinary projects, which went on to produce numerous installations and performances featuring elements of interaction, robotics and telecommunications. Since 1995, Gerfried Stocker has been artistic director of Ars Electronica. In 1995-96, he headed the crew of artists and technicians that developed the Ars Electronica Center's pioneering new exhibition strategies and set up the facility's in-house R\&D department, the Ars Electronica Futurelab. He has been chiefly responsible for conceiving and implementing the series of international exhibitions that Ars Electronica has staged since 2004, and, beginning in 2005, for the planning and thematic repositioning of the new, expanded Ars Electronica Center. 